\title{
The discrimination of heading from optic flow is not retinally invariant
}

\author{
PAUL ATCHLEY \\ University of Illinois, Urbana-Champaign, Illinois \\ and \\ GEORGE J. ANDERSEN \\ University of California, Riverside, California
}

\begin{abstract}
Three experiments were conducted to determine whether the discrimination of heading from optic flow is retinally invariant and to determine the importance of acuity in accounting for heading eccentricity effects. In the first experiment, observers were presented with radial flow fields simulating forward translation through a three-dimensional volume of dots. The flow fields subtended $10^{\circ}$ of visual angle and were presented at $0^{\circ}, 10^{\circ}, 20^{\circ}$, and $40^{\circ}$ of retinal eccentricity. The observers were asked to indicate whether the simulated movement was to the right or the left of a target that appeared at the end of the display sequence. Eye movements were monitored with an electrooculogram apparatus. In a second experiment, static acuity thresholds were derived for each of the observers at the same retinal eccentricities. There was a significant increase in heading detection thresholds with retinal eccentricity (from $0.92^{\circ}$ at $0^{\circ}$ retinal eccentricity to $3.47^{\circ}$ at $40^{\circ}$ ). An analysis of covariance indicated that the variation in sensitivity to radial flow, as a function of retinal eccentricity, is independent of acuity. Similar results were obtained when the Vernier acuity of observers was measured. These results suggest that the discrimination of heading from radial flow is not retinally invariant.
\end{abstract}

A perceptual task of critical importance for successful navigation is to accurately determine and control the direction of observer motion. One source of information that can be used to determine the direction of observer motion - or heading - is optic flow (see Figure 1). Gibson $(1950,1966)$ proposed the term optic flow to describe the transformations of the optic array during motion of the observer. The focus of expansion (FOE; Gibson, 1966) is a singularity of the optic flow field that, it has been suggested, can be detected primarily by the central visual field. The FOE is the point from which, during forward translation through the visual environment, optic flow originates and at which image velocities are closest to zero. The FOE has been demonstrated to be an important source of information for the discrimination of heading (e.g., Warren \& Hannon, 1990). However, other sources of information, such as differential motion parallax (DMP; Cutting, Springer, Braren, \& Johnson, 1992) or the global properties of the optic flow fields (Dyre \& Andersen, 1997), have been shown to be useful as well.

An important area of research that has had a significant impact on theories of heading sensitivity is studies

This research was partially supported by an AFOSR Fellowship to the first author and Grants SBR 95-10431 and AG13419-01. The authors thank John Cisneros for assistance in the development of the experiments. Correspondence concerning this article should be sent to G. J. Andersen, Department of Psychology, University of California, Riverside,CA 92521 (e-mail: andersen@citrus.ucr.edu). concerned with vection - the perception of self-motion. Early research on the perception of self-motion argued that the visual system was primarily sensitive to information about self-motion in the retinal periphery (a greater than $30^{\circ}$ diameter area around the fovea). In addition, these studies suggested that the central visual field (a within $30^{\circ}$ diameter area surrounding the fovea) was responsible for determining object motion. This hypothesis, originally referred to as the focal/ambient theory (Held, 1970), has also been referred to as the peripheral dominance hypothesis (Held, Dichgans, \& Bauer, 1975 ). Held et al. argued that the peripheral visual field dominated the central visual field in maintaining spatial orientation and in producing a perception of self-motion (see, e.g., Brandt, Dichgans, \& Koenig, 1973). Evidence against this hypothesis was found in studies demonstrating that self-motion (Andersen \& Braunstein, 1985) and postural sway (Andersen \& Dyre, 1989) from optic flow could occur from stimulation of the central visual field. Andersen and Braunstein proposed that the visual system could process information throughout the visual field, but that information for determining self-motion differed across the visual field. Evidence in support of this hypothesis can be found in studies concerned with postural adjustments (Stoffregen, 1985; Stoffregen \& Riccio, 1990).

It is important to note that expansion information that specifies heading falls on the central portion of the retina under most conditions of forward translation. This observation suggests that the central portion of the retina should 


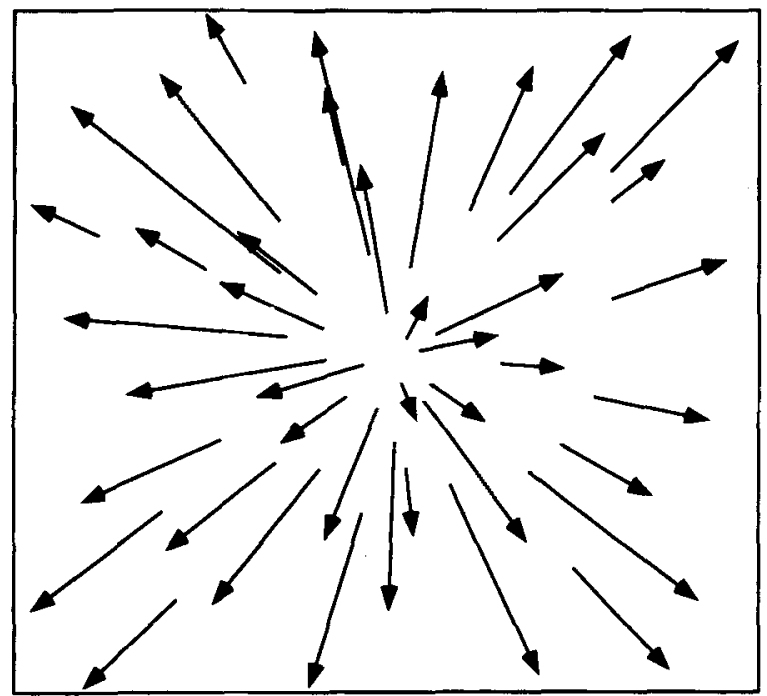

Figure 1. Illustration of a radial-expanding optic flow field of the type examined in the present study, simulating observer motion through a three-dimensional volume of points.

be more sensitive to this type of information and, therefore, able to more accurately detect heading than can more peripheral parts of the retina, when expansion information is present. This possibility has been called the functional sensitivity hypothesis (Stoffregen, 1985; Warren \& Kurtz, 1992). Warren and Kurtz performed a series of experiments in which the functional sensitivity hypothesis was examined. In one experiment, observers were asked to indicate their heading, relative to a fixation cross. Three viewing conditions-full field of view (FOV), central FOV, or peripheral FOV-were used. The sizes of the central and peripheral visual fields were varied by masking either the outer (for the central FOV) or the inner (peripheral FOV) regions of the flow field. The results indicate that decreasing the outer region of the flow field resulted in little change in heading accuracy. However, masking the inner region resulted in a decrease in heading accuracy. In a second experiment, the retinal location of the FOE was varied. As the FOE became more eccentric (from $0^{\circ}$ to $15^{\circ}$ ), performance decreased. Their results suggest that the central visual field is more sensitive to radial flow information for determining the heading.

Crowell and Banks (1993) noted several problems with the study by Warren and Kurtz (1992) that limit their conclusions. For example, Crowell and Banks (1993) noted that, in the first experiment of Warren and Kurtz, the location of radial flow covaried with retinal location. They also argued that, in the second experiment, retinal position covaried with the pattern of flow. Crowell and Banks (1993) conducted an experiment to test whether sensitivity to radial flow was retinally invariant (the retinal invariance hypothesis), by presenting two heading displays in succession and asking the observers to indicate whether the heading direction in the second interval was to the left or the right of the heading direction in the first interval.
Heading eccentricity (the angle between the specified heading and the center of the stimulus) varied from $0^{\circ}$ to $70^{\circ}$, and retinal eccentricity (the angle between the fovea and the center of the stimulus) varied from $0^{\circ}$ to $40^{\circ}$. The results indicated that judgments were more accurate with radial flow fields (small heading eccentricities) than with lamellar flow fields (large heading eccentricities). However, they also report that "retinal eccentricity effects in heading judgments are relatively small" (p. 332), arguing that there is a "fairly small and inconsistent effect of retinal eccentricity on the ability to perceive heading" (p. 333). They concluded that "peripheral vision ... is as efficient as central vision with all types of flow fields except radial ones, with which it is somewhat less efficient" (p. 335) and that "the visual system is well adapted for extracting the information specifying heading in a variety of patterns of optic flow and from a variety of retinal regions" (p. 336). The results and conclusions of the Crowell and Banks (1993) study have been interpreted as indicating that there is no retinal specialization for radial flow patterns (Warren, 1995) and that the perception of heading is largely independent of retinal position (Cutting, Vishton, Flückiger, Baumberger, \& Gerndt, 1997).

Finally, in recent work by Cutting et al. (1997), the role of retinal eccentricity in the detection of heading was examined with displays simulating smooth pursuit. Observers were presented with stimuli simulating observer motion through a three-dimensional (3-D) environment consisting of trees. A red fixation tree was located at the center of the display. In one set of displays, environments were viewed through circular apertures in the retinal periphery of various radii. In a second set of displays, the central region of the display was masked. The results indicated that accuracy in detecting heading decreased for both conditions when the area of stimulation was reduced. To examine the role of retinal eccentricity, they compared the performance across both peripheral aperture and central mask conditions. Variations in acuity and motion sensitivity, as a function of retinal eccentricity, were controlled for by scaling the data with the results from previous studies on acuity and motion sensitivity. A rescaling of the data produced identical performance for both peripheral aperture and central mask conditions across variations in retinal eccentricity. According to Cutting et al. (1997), the results provide evidence in support of a retinal sensitivity hypothesis in which accuracy in heading, as a function of the stimulation of different retinal eccentricities, is dependent on variations in motion sensitivity across the visual field.

The purpose of the present study was twofold. Its first purpose was to clarify the question of whether sensitivity to radial flow is retinally invariant. Warren and Kurtz (1992) reported a large effect of retinal eccentricity. However, there were several problems with the study, as was noted by Crowell and Banks (1993). Crowell and Banks (1993) argued that there was a small, inconsistent effect of retinal eccentricity. However, in recent papers reviewing the results of Crowell and Banks (1993), it is argued 
that the paper is evidence in support of the retinal invariance hypothesis. Finally, recent research by Cutting et al. (1997) suggests that sensitivity to heading information, as a function of eccentricity, is dependent on sensitivity to motion at different parts of the retina. In summary, there does not appear to be a clear consensus in the literature regarding the effect of retinal eccentricity on the perception of heading.

The lack of a consensus is further complicated by the different methodologies that have been used. In the study by Cutting et al. (1997), the stimuli simulated pursuit fixation. In the experiment of Crowell and Banks (1993), observers were required to make comparison judgments of two optic flow displays. However, the study by Warren and Kurtz (1992) required observers to determine heading relative to a target-a task used in the vast majority of heading research (e.g., Cutting, 1986, 1996; Cutting et al., 1992; Dyre \& Andersen, 1997; Warren \& Hannon, 1990; Warren, Morris, \& Kalish, 1988). It is possible that determining whether a second flow field has a heading direction to the left or the right of a previously presented flow field may not reflect the performance of observers in heading tasks when the judgment is relative to a target - a task likely to be important in real-world heading tasks involving navigation and collision avoidance.

To address this issue, we presented observers with optic flow displays in which they were required to judge whether they were heading to the left or to the right of a target. We also varied the retinal eccentricity at which the displays were viewed. Finally, eye movements were measured, to ensure that observers maintained fixation and that flow fields were presented to the appropriate regions of the visual field.

The second purpose of the present study was to investigate the role of acuity in the discrimination of heading when retinal eccentricity is varied. It is possible that detecting heading for stimuli presented in different regions of the visual field would be limited by the spatial resolution of the visual system. In the Cutting et al. (1997) study, acuity sensitivity was considered by scaling the heading performance to previously published data. An ideal method for addressing this issue is to measure these thresholds for each individual observer, so that each observer serves as his or her own control. To address this issue, we measured the visual acuity of observers, using either a Landolt $C$ stimulus (Experiment 2) or a Vernier acuity stimulus (Experiment 3 ). By measuring the acuity of the observers, we were able to determine the effects of retinal eccentricity, independently of acuity, on heading thresholds.

\section{EXPERIMENT 1}

\section{Method}

Observers. Twenty students (10 men and 10 women with a mean age of 22.7 years) from the University of California, Riverside, participated in this study and were paid for their participation. The average (corrected) Snellen static acuity was $20 / 20.2$ for the right eye.

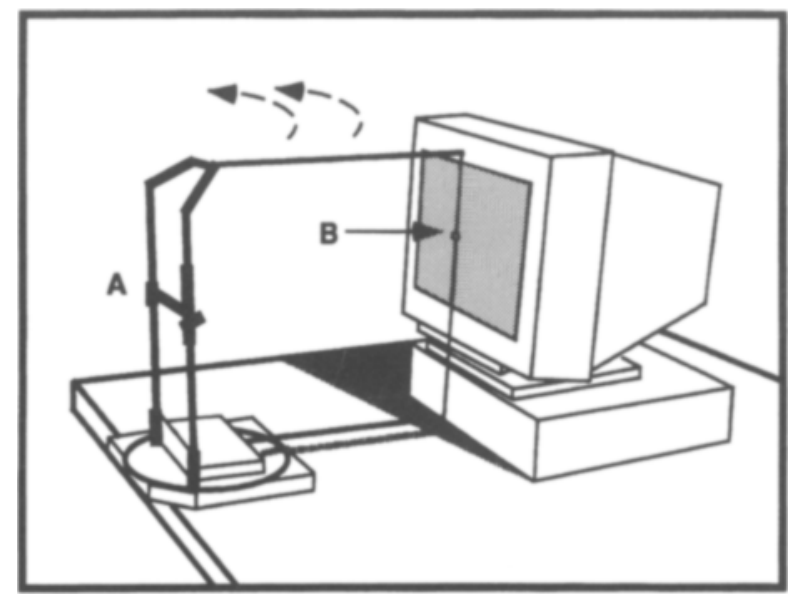

Figure 2. Illustration of the head mount and fixation apparatus used in the present study.

Apparatus and Stimuli. A Silicon Graphics (SGI) Indigo2 Extreme computer system was used to generate the displays. The displays were presented at a distance of $52.07 \mathrm{~cm}$ on a $53.34-\mathrm{cm} \mathrm{SGI}$ monitor with a refresh rate of $72 \mathrm{~Hz}$. The observer's head was stabilized with a headmount (see Figure 2). The headmount rotated up to the maximum retinal eccentricity $\left(40^{\circ}\right)$ and locked at each one of the four viewing angles $\left(0^{\circ}, 10^{\circ}, 20^{\circ}\right.$, or $\left.40^{\circ}\right)$. A red light-emitting diode (LED) was attached to the headmount at the same distance as the display and served as the fixation point in this experiment. Changes in retinal eccentricity were produced by rotating the headmount and fixation point (and thereby moving the observer's head), rather than by moving the display.

To control for eye movements, an electrooculogram (EOG) was used to monitor the fixation position of the observer for each trial. Horizontal EOG was recorded from two electrodes located laterally to each eye. The electrodes were $\mathrm{Ag} / \mathrm{AgCl}$ in composition and were attached with an earphone-like apparatus and electrode cream (Neuromedical Supply, Inc.). An earclip was attached to the ear to provide the ground for the system. The EOG signal was amplified by a Grass $12 \mathrm{~A} 5$ amplifier and passed to an oscilloscope, where changes in eye position were displayed as changes in the position of a horizontal bar. The resolution of this apparatus was $2^{\circ}$ of deflection from fixation.

Each display consisted of a radial flow display with 200 points. Display duration was $2 \mathrm{sec}$. The background and point luminances were $0.021 \mathrm{~cd} / \mathrm{m}^{2}$ and $2.83 \mathrm{~cd} / \mathrm{m}^{2}$, respectively. The displays subtended a $10^{\circ}$ circular region and simulated a 3-D space with an extent in depth of $1.65 \mathrm{~m}$ at an eye distance of $0.2 \mathrm{~m}$. The displays simulated forward translation through a cloud of dots at either $0.41 \mathrm{~m} / \mathrm{sec}$ or $1.64 \mathrm{~m} / \mathrm{sec}$. Pixel size was 1.4 minarc and was constant across all simulated depths. The angle of forward translation through the simulated 3-D environment was manipulated as a function of an adaptive staircase procedure and always started $5^{\circ}$ to the left or the right of the center of the viewing region. Four retinal eccentricities $\left(0^{\circ}\right.$, $10^{\circ}, 20^{\circ}$, and $40^{\circ}$ ) and two stimulus velocities (simulated forward speeds of $0.41 \mathrm{~m} / \mathrm{sec}$ and $1.64 \mathrm{~m} / \mathrm{sec}$ ) were used. The target line subtended $5^{\circ}$ of visual angle. An eyepatch was used to cover the left eye. The stimulus parameters were held constant over the entire range of retinal eccentricities tested.

Procedure. After the EOG apparatus and the eyepatch were attached, the eye movement system was calibrated by having the observer make eye movements from one edge of the screen to the other edge. Following this procedure, the observer was dark adapted for a period of $5 \mathrm{~min}$ while the experimenter read the instructions and explained the task. The observer was then shown displays of the two 


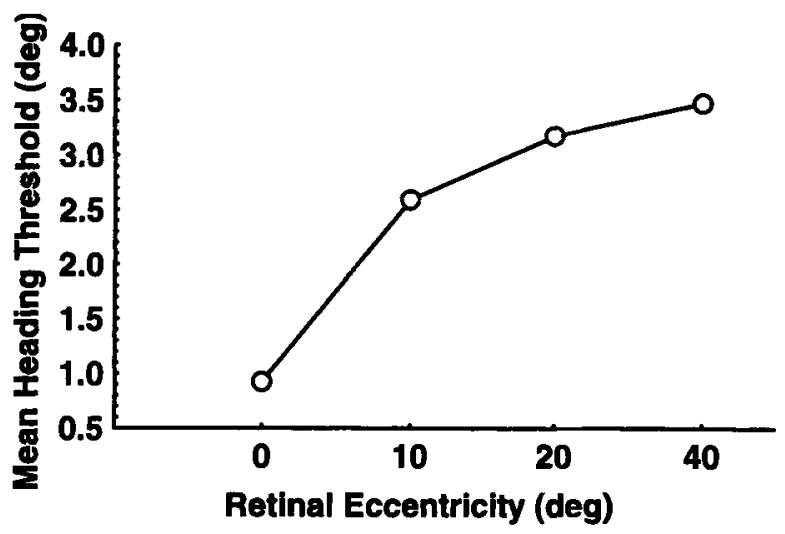

Figure 3. Mean heading thresholds as a function of retinal eccentricity.

velocities at a viewing angle of $0^{\circ}$. The observer was instructed to fixate the red LED at all times during the experimental trials, even when the target and the LED were not in the same location (i.e., the conditions in which the retinal eccentricity was not $0^{\circ}$ ). The observer was then positioned at the appropriate retinal eccentricity (viewing order was randomized for each observer). While the display was on the screen, the experimenter monitored the eye positions of the observer, to ensure that he or she was properly fixated and had not shifted his or her gaze to the display. An observer who failed to maintain fixation was reminded to fixate only on the red LED. Trials on which the observer failed to fixate were repeated.

The observer's task was to judge the heading relative to the center of the motion display. The display simulated translation through a cloud of dots at a specified heading angle, relative to forward translation along the line of sight (for an observer directly facing the display). At the end of each trial, a target bar appeared, to indicate the location of the center of the display. ${ }^{1}$ The task of the observer was to judge the direction of translation relative to the marker line (to the right or to the left) and to verbally indicate the direction to the experimenter. The response of the observer was recorded by computer.

Thresholds were derived on the basis of a two-down/one-up adaptive staircase procedure. After two correct judgments in a row, the heading angle decreased by $50 \%$. After one error, it increased by $50 \%$. Two runs were conducted for each condition. Each run continued for 10 reversals, with a preliminary threshold being determined by the average of the last 4 reversals. The average of two preliminary thresholds (one threshold for each run) for each combination of retinal eccentricity and simulated speed was used to define a final threshold. The observer participated in four separate blocks-one block for each retinal eccentricity. The order of the blocks was randomized across observers. All the trials were completed monocularly with the right eye. The experiment took approximately $1 \mathrm{~h}$ to complete.

\section{Results}

A $2 \times 4$ (flow rate $\times$ retinal eccentricity) analysis of variance (ANOVA) was performed on the heading thresholds for each observer for each condition. There was a significant main effect for retinal eccentricity $[F(3,57)=$ $11.90, p<.001]$. The effect of eccentricity on heading thresholds is depicted in Figure 3. According to this result, heading thresholds increased as retinal eccentricity increased. The average thresholds for the $0^{\circ}, 10^{\circ}, 20^{\circ}$, and $40^{\circ}$ retinal eccentricities were $0.92,2.58,3.17$, and
3.47 , respectively. A post hoc comparison of the thresholds with a Tukey HSD analysis $[q p<.05(3,57)=3.41]$ revealed that performance at $0^{\circ}$ of retinal eccentricity was lower than that in the $10^{\circ}, 20^{\circ}$, and $40^{\circ}$ conditions. No other pairwise comparisons were significant. The effect of flow rate was not significant $[F(1,19)=0.90, \mathrm{n} . \mathrm{s}$.] The interaction of retinal eccentricity and flow rate also was not significant $[F(3,57)=0.52$, n.s. $]$.

\section{Discussion}

The results of the first experiment indicate that the discrimination of heading from optic flow-as measured with regard to a target - is not retinally invariant. Heading detection thresholds increased significantly when optic flow fields were presented beyond $0^{\circ}$ of retinal eccentricity (from $0.92^{\circ}$ at $0^{\circ}$ of retinal eccentricity to $3.47^{\circ}$ at $40^{\circ}$ of retinal eccentricity).

\section{CONTROL EXPERIMENT}

One potential problem with the methodology used in Experiment 1 is the presence of the fixation point in the central vision $\left(0^{\circ}\right)$ condition (see note 1$)$. In this condition, the fixation LED was presented at the center of the display throughout the trial. The position of the LED was coincident with the location of the target bar that appeared at the end of the trial. Thus, the presence of the LED provided a reference against which local dot motion could be compared. Because this information was available only in the $0^{\circ}$ viewing eccentricity condition, it may have provided additional information that was useful to the observer in determining heading direction. This observation suggests that the improved performance of the $0^{\circ}$ eccentricity condition may have been the result of the motion of the dots relative to the fixation point, rather than of greater sensitivity to radial flow information in the central visual field.

To address this issue, we conducted a control experiment in which sensitivity to radial flow in central vision was examined. If the presence of a fixation point provides information useful for determining heading, performance should be greater when a fixation point is present than when a fixation point is absent. To test this hypothesis, we replicated the central field condition of the first experiment and varied whether or not a fixation point was present.

\section{Method}

Observers. Eight students ( 5 men and 3 women) from the University of California, Riverside, participated in this study and were paid for their participation. All of the observers were naive with regard to the purpose of the study. The average (corrected) Snellen static acuity was $20 / 20.3$ for the right eye.

Apparatus and Stimuli. The apparatus and stimuli were identical to those used in Experiment 1.

Procedure. The procedure was identical to that in Experiment 1, with the following exception. Only the $0^{\circ}$ retinal eccentricity was examined. Heading thresholds were derived for two conditionswith and without the fixation point. The order of the fixation point manipulation was counterbalanced across observers. When the fix- 
ation point was present, the observers were instructed to fixate the LED. The instructions to the observers when the fixation point was present were identical to the instructions in Experiment 1 . When the fixation point was absent, the instructions were identical to those used in Experiment 1, with the exception that they were asked to stare at the center of the display.

\section{Results}

A $2 \times 2$ (flow rate $\times$ fixation condition) ANOVA was performed on the heading thresholds for each observer for each condition. The main effect of fixation condition was significant $[F(1,7)=7.37, p<.05]$. According to this effect, heading thresholds were lower for the fixationabsent condition (mean heading thresholds of $0.71^{\circ}$ ) than for the fixation-present condition (mean heading thresholds of $1.26^{\circ}$ ). There were no other significant main effects or interactions.

\section{Discussion}

The results of the present experiment indicate that heading thresholds were higher when the fixation point was present. These results suggest that the presence of a fixation point did not provide information that was useful in detecting heading.

\section{EXPERIMENT 2}

One possible explanation for the results of Experiment 1 is that the ability of observers to use the FOE in the most retinally eccentric cases is limited by the spatial resolution of the peripheral visual system. Specifically, heading detection thresholds may be higher in the peripheral field of view because of reduced acuity in locating the FOE relative to the target line. Previous studies have demonstrated that the ability to spatially resolve position information is reduced in the retinal periphery (Levi, Klein, \& Aitsebaomo, 1985). This issue was a factor that led Crowell and Banks (1993) to use a paradigm in which observers compared two flow fields presented sequentially, rather than one in which they made a heading judgment relative to a target.

To examine this possibility, a second experiment was conducted, in which the static acuity thresholds were derived for each observer for each of the retinal eccentricity locations tested in Experiment 1. If the results of Experiment 1 are due to acuity differences, we would expect a significant degree of correlation between the heading and the acuity thresholds of the observers.

In this experiment, we derived static acuity thresholds for each of the observers who participated in the first experiment for each of the retinal eccentricity values tested. The same apparatus as that used in the first experiment was used in this experiment. Acuity thresholds were derived with a modified Landolt $C$ test in which the target stimulus maintained a fixed size and the size of the gap of the Landolt $\mathrm{C}$ was varied.

\section{Method}

Observers. The same 20 observers who participated in the first experiment participated in this experiment.
Apparatus and Stimuli. The apparatus was the same as that used in the first experiment. The stimulus used to derive acuity thresholds was a Landolt $\mathrm{C}$. The Landolt consisted of a white $\mathrm{C}$ on a black surround and subtended $5^{\circ}$ of visual angle for all the viewing eccentricities. The gap in the C could appear in one of four positions: upper left, upper right, lower left, or lower right (Brown, 1972). The duration of the target was $500 \mathrm{msec}$. The size of the gap started at $3^{\circ}$ of visual angle. The gap size could be varied in 2 -minarc increments. All the trials were completed monocularly with the right eye. An eyepatch was used to cover the left eye.

Procedure. After the EOG apparatus and the eyepatch were attached, the observer was dark adapted for a period of 5 min while the experimenter read the instructions and explained the task. The observer was then shown a demonstration of the displays at a viewing angle of $0^{\circ}$. The observer was instructed to fixate on the red LED at all times during the experimental trials, even when the target and the LED were not in the same location (i.e., the conditions in which the retinal eccentricity was not $0^{\circ}$ ). The observer's task was to verbally report the location of the gap in the Landolt $\mathrm{C}$ by indicating upper right, lower right, upper left, or lower left. The observer was instructed to make his or her best guess, if he or she was unable to detect the gap.

The experimental trials were started after the instruction phase. At the beginning of each assessment series, an "X" appeared on the screen and flashed three times, at a rate of $1 \mathrm{~Hz}$, at the location at which the Landolt $C$ would initially appear. The " $X$ " also appeared (without flashing) in the same location at the start of each trial and remained until the experimenter started the trial. The " $X$ " served to orient the attention of the observer to the display. While the orientation point was on the screen, the experimenter monitored the eye position of the observer, to ensure they were properly fixated and had not shifted their gaze to the orientation point. Observers who failed to maintain fixation were reminded to fixate only on the red LED. When the observer was properly fixated, the experimenter initiated presentation of the Landolt $C$. The "X" was replaced with a blank screen for $1 \mathrm{sec}$, followed by the presentation of the Landolt C. After the Landolt $\mathrm{C}$ disappeared, the observer indicated the orientation of the gap. This response was recorded via computer by the experimenter.

The order of retinal eccentricity was randomized across observers. Thresholds were derived for all observers for all retinal eccentricities. A two-down/one-up adaptive staircase was used to derive the acuity thresholds. Two correct responses were followed by a decrease in the gap size by $50 \%$. An incorrect response was followed by an increase in the gap size by $50 \%$. A change from a correct response to an incorrect response or from an incorrect response to a correct response was counted as a reversal. An assessment series was concluded after eight reversals or if the minimum resolution ( 2 minarc) was reached. Each observer participated in two runs of the adaptive staircase procedure. A preliminary threshold was derived on the basis of the average of the last four reversals of a run. The average of the two preliminary thresholds was taken as the acuity value for that retinal eccentricity.

\section{Results}

A one-way within-observers ANOVA was used to determine whether there was a significant decrease in acuity with increasing retinal eccentricity. The results of the ANOVA revealed a significant effect of retinal eccentricity $[F(3,57)=27.96, p<.001$; see Figure 4$]$. A post hoc comparison of the thresholds with a Tukey HSD analysis $[q p<.05(3,57)=3.41]$ revealed that thresholds at $40^{\circ}$ of retinal eccentricity ( 33.7 minarc) were significantly different from those obtained at all the other retinal eccentricities $\left(2.3\right.$ minarc at $0^{\circ}, 10.4$ minarc at $10^{\circ}$, and 10.6 minarc at $20^{\circ}$ ). No other differences were significant. 


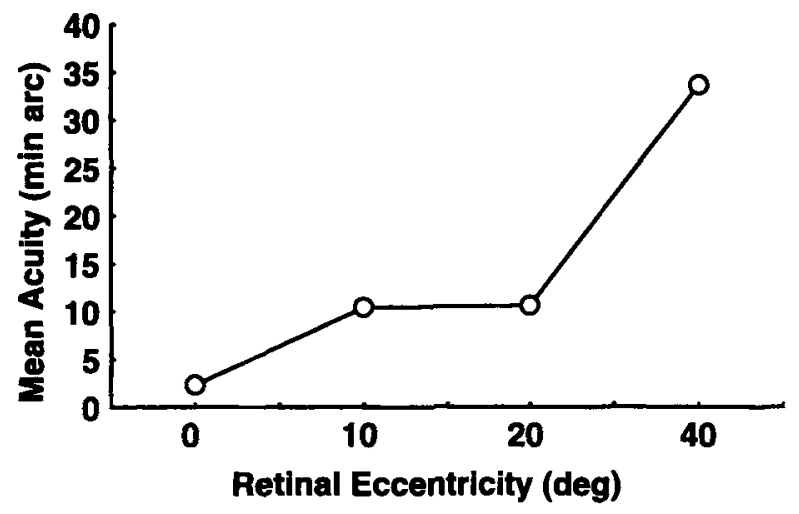

Figure 4. Mean acuity thresholds as a function of retinal eccentricity.

The acuity thresholds for each observer were then correlated with their heading detection thresholds from the first experiment. None of the correlations between the observers acuity and heading detection thresholds reached significance $(p>.05)$ for any of the retinal eccentricity conditions examined. The Pearson $r$ values for the $0^{\circ}, 10^{\circ}$, $20^{\circ}$, and $40^{\circ}$ retinal eccentricities were $-.04,-.19, .08$, and .17 , respectively. These correlations indicate that acuity thresholds at each eccentricity were not correlated with heading thresholds at each eccentricity level.

A second correlation analysis was conducted to determine whether acuity thresholds, across levels of retinal eccentricity, were correlated with heading thresholds. The Pearson $r$ correlation $(r=.33)$ was significant $[t(78)=$ $3.19, p<.05]$, accounting for $11 \%$ of the variability. This result suggests that static acuity accounts for some of the variablity for the significant effect of eccentricity on heading thresholds observed in Experiment 1 . In order to determine the effect of retinal eccentricity independent of acuity, an analysis of covariance (ANCOVA) was conducted in which the variability owing to acuity was partitioned out of the ANOVA (see Keppel, 1982, for a discussion of this analysis). The post hoc analysis of the main effect of retinal eccentricity in Experiment 1 indicated that the $0^{\circ}$ heading thresholds were significantly lower than the $10^{\circ}, 20^{\circ}$, and $40^{\circ}$ heading thresholds. Therefore, we conducted an ANCOVA for each of these pairwise comparisons, to determine whether the differences observed in Experiment 1 were significant independent of acuity.

The ANCOVA results indicated that all three comparisons were significant. The $F$ ratios and significance levels for the $0^{\circ}$ and $10^{\circ}$ comparison, the $0^{\circ}$ and $20^{\circ}$ comparison, and the $0^{\circ}$ and $40^{\circ}$ comparison were $F(1,18)=12.36$ $(p<.002), F(1,18)=6.37(p<.02)$, and $F(1,18)=18.37$ $(p<.001)$, respectively. Thus, the results indicate that sensitivity to radial flow in the central field - when considered independent of acuity-is greater than the sensitivity in more peripheral regions of the visual field. The $\omega^{2}$ for these comparisons were $.36, .21$, and .38 , respectively. Cohen (1977) has proposed that effect size in the behav- ioral sciences greater than .15 can be categorized as a large effect. These results indicate that the sensitivity to radial flow information for determining heading - when analyzed independent of acuity - varies as a function of retinal eccentricity and that the variance accounted for in these results can be categorized as large effects.

\section{EXPERIMENT 3}

The results of Experiment 2 indicate that the increases in heading thresholds observed in Experiment 1 were not due to changes in acuity as these were measured with a Landolt $\mathrm{C}$ target. An important question, however, is whether other measures of acuity might be more appropriate for assessing the role of acuity in the heading task in Experiment 1. For example, the heading thresholds derived in Experiment 1 were measured by asking the observers to judge the heading relative to a target bar that was presented after the heading stimulus - a task based on comparing two locations. This observation suggests that a Vernier acuity measure, which requires observers to judge the spatial displacement of two locations, might be more appropriate than a Landolt $\mathrm{C}$ measure. In addition, the heading task involves a comparison of two locations that are presented sequentially, rather than simultaneously. This suggests that the presentation of two positions in the acuity measure should be offset temporally.

To address this issue, we conducted the following experiment. The experiment consisted of two phases involving judgments by the same group of observers. The first phase involved measuring heading thresholds by replicating the conditions examined in Experiment 1. The second phase involved measuring Vernier acuity for each retinal eccentricity examined in Experiment 1 . To measure Vernier acuity, we used a two-interval forced-choice paradigm in which observers were sequentially presented with two displays, each containing two vertical line segments. In one display, the vertical line segments were spatially offset, whereas in the other display, the vertical line segments formed a single line. The task of the observer was to indicate which of the two displays contained the offset.

In addition, within each display, two line segments were sequentially presented. The duration and time course of the presentation for each display was identical to the time course and presentation of the flow field and target bar used to determine heading thresholds. We then conducted an ANCOVA to factor out the Vernier acuity of each observer, to determine whether sensitivity to radial flow varied as a function of eccentricity independent of acuity.

\section{Method}

Observers. Seven students ( 4 men and 3 women) from the University of California, Riverside, participated in this study and were paid for their participation. All of the observers were naive with regard to the purpose of the study and had previously participated in the control experiment. 
Apparatus. The apparatus was identical to that used in Experiment 1 .

Heading threshold stimuli. The stimuli for determining heading thresholds were identical to those used in Experiment 1 .

Vernier acuity stimuli. The stimuli consisted of two vertical lines ( 1.4 minarc by $5^{\circ}$ ). Within each trial, two different stimuli were presented, which we will refer to as the aligned and the offset stimuli. The aligned stimulus consisted of two vertical lines that were aligned to produce a single continuous line. The offset stimulus consisted of two vertical lines that were slightly offset to the left and the right of the position of the single continuous line. The direction of offset of the two vertical lines was randomized across trials. The angle of offset was manipulated as a function of an adaptive staircase procedure and always started at $5^{\circ}$. For the $0^{\circ}$ eccentricity condition, the vertical lines were positioned relative to a central position (the position of the aligned stimulus) that was $0.1^{\circ}$ to the right of the center of the display. This was done to prevent a spatial overlap with the fixation LED for this condition. Thus, the horizontal location of the aligned stimulus was $25^{\circ}, 10^{\circ}, 20^{\circ}$, or $40^{\circ}$ of retinal eccentricity.

Each trial consisted of the presentation of a stimulus, followed by a 5-sec period, followed by the presentation of a second stimulus. Within each display, the two line segments were presented sequentially. Each segment was presented for 2 sec.

General procedure. The observers participated in two separate phases of the experiment. During the first phase, heading thresholds were derived, whereas during the second phase, acuity thresholds were derived. Within each phase, the observers participated in four separate blocks - one block for each retinal eccentricity. The order of the blocks was randomized across observers.

Heading procedure. The procedure was identical to that in Experiment 1 .

Vernier acuity procedure. The procedure was identical to that in Experiment 1, with the following exceptions. A two-interval forced-choice procedure was used. The observers were instructed that they would be presented two displays sequentially. Each display contained two line segments that were presented one after the other. In one display, the line segments were perfectly aligned to form a single line. In the other display, the line segments were offset horizontally. The task of the observer was to indicate which of the two displays contained the horizontally offset line segments.

Thresholds were derived with a two-down/one-up adaptive staircase procedure identical to that in Experiment 1.

\section{Results}

Heading thresholds. A $2 \times 4$ (flow rate $\times$ retinal eccentricity) ANOVA was performed on the heading thresholds for each observer for each condition. There was a significant main effect for retinal eccentricity $[F(3,18)=$ $6.06, p<.01]$. The average thresholds for the $0^{\circ}, 10^{\circ}$, $20^{\circ}$, and $40^{\circ}$ retinal eccentricities were $0.86,2.57,2.92$, and 3.22 , respectively. A post hoc comparison of the thresholds, using a Tukey HSD analysis, revealed that performance at $0^{\circ}$ of retinal eccentricity was lower than the $10^{\circ}, 20^{\circ}$, and $40^{\circ}$ conditions. No other pairwise comparisons were significant. There were no other significant main effects or interactions. These results replicate the effect of eccentricity observed in Experiment 1.

Acuity thresholds. A one-way (retinal eccentricity) ANOVA was performed on the acuity thresholds for each observer for each condition. There was a significant main effect for retinal eccentricity $[F(3,18)=9.35, p<$ .011 . The average thresholds for the $0^{\circ}, 10^{\circ}, 20^{\circ}$, and $40^{\circ}$ retinal eccentricities were $0.02,0.21,0.30$, and 0.59 , respectively. A post hoc comparison of the thresholds, using a Tukey HSD analysis, revealed that performances at the $0^{\circ}$ and $10^{\circ}$ conditions were lower than the thresholds for the $40^{\circ}$ conditions. No other pairwise comparisons were significant. This result replicates the effect of eccentricity observed in Experiment 2, using a Landolt C stimulus.

ANCOVA. In order to determine the effect of retinal eccentricity as independent of acuity, an ANCOVA was conducted by controlling for the acuity of each subject. The post hoc analysis of the main effect of eccentricity for heading thresholds indicated significant differences between the $0^{\circ}$ and $40^{\circ}$ and the $10^{\circ}$ and $40^{\circ}$ conditions. Therefore, we conducted an ANCOVA for both of these pairwise comparisons. The $F$ ratios and significance levels for the $0^{\circ}$ and $40^{\circ}$ comparison and the $10^{\circ}$ and $40^{\circ}$ comparison were $F(1,6)=16.1, p<.006$, and $F(1,6)=11.6$, $p<.01$, respectively. These results indicate that sensitivity to radial flow information for determining heading varies as a function of retinal eccentricity independent of Vernier acuity thresholds.

\section{MOTION SENSITIVITY AND RETINAL ECCENTRICITY}

In the present study, we have examined the retinal invariance hypothesis by factoring out variability of each observer, using acuity measures. These analyses allowed us to assess the sensitivity to radial flow in heading judgments independent of retinal acuity. An alternative issue is to consider the sensitivity to motion in heading judgments. Cutting et al. (1997) examined this issue by collecting heading judgments when motion information was presented to different regions of the visual field and rescaling the data, using results from Leibowitz, Johnson, and Isabelle (1972). To rescale the data, Cutting and his colleagues calculated values at each retinal eccentricity examined by normalizing motion sensitivity across the visual field, relative to sensitivity in the central visual field $\left(0^{\circ}\right)$. It is important to note that the study by Leibowitz et al. examined motion sensitivity in detecting the direction of motion of a square. Ideally, one would want to compare heading performance with variations in sensitivity to radial motion as a function of retinal location.

Recently, Atchley and Andersen (1998) examined this issue by measuring differences between older and younger observers in sensitivity to optic flow components (including radial motion) at different retinal eccentricities. The observers were presented with radial flow fields in which the coherence of the motion pattern was adjusted until it was no longer detectable. With this paradigm, motion coherence thresholds were derived for radial flow at $0^{\circ}, 10^{\circ}, 20^{\circ}$, and $40^{\circ}$ of eccentricity. The simulated speed of observer motion was identical to the speeds used in Experiment 1. The average results for 20 college-age observers are presented in Figure 5, along with the data from Experiments 1 and 3. As is shown in Figure 5, the graph for sensitivity to radial flow is re- 

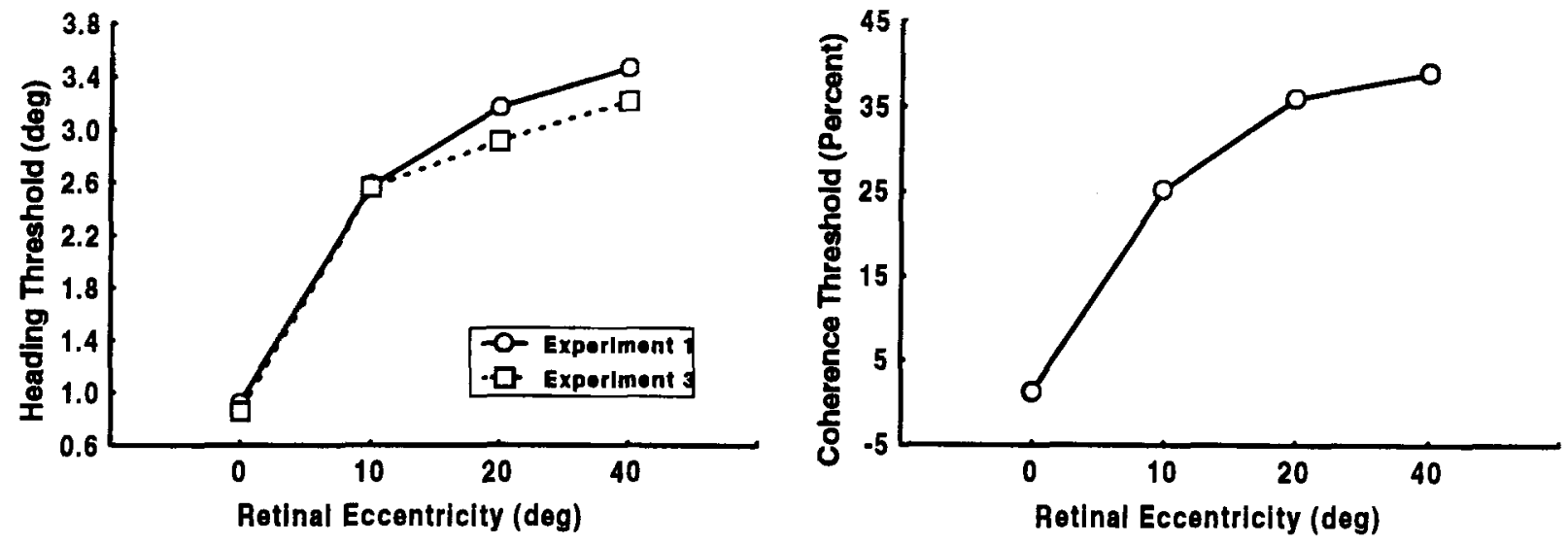

Figure 5. Graphs of motion sensitivity and heading sensitivity as a function of retinal eccentricity. The graph on the left represents the results of Experiments 1 and 3. The graph on the right is from Atchley and Andersen (1998), in which motion coherence thresholds were derived for radial motion.

markably similar to the heading thresholds observed in Experiments 1 and 3. Indeed, the correlation between heading detection performance and coherence thresholds for radial motion is $r=1.00$ for Experiment 1 and $r=.99$ for Experiment 3 . Both of these correlations are significant $(p<.01)$. These results suggest that variations in sensitivity to radial flow, across the visual field, are highly predictive of the variations in heading thresholds at different retinal eccentricities.

\section{GENERAL DISCUSSION}

The results of the present study indicate that determining the direction of observer motion from radial flow varies as a function of retinal eccentricity - with greater sensitivity in the central field than in the retinal periphery. In the first experiment, we presented the observers with optic flow displays in which they were required to judge whether the heading direction was to the left or the right of a target line. Retinal eccentricity was varied to determine whether heading thresholds for radial flow varied as a function of retinal eccentricity. We found that heading detection thresholds for peripheral portions of the visual field were up to $250 \%$ higher than those for central vision. In a second experiment, we examined the spatial resolution of the visual system at the retinal eccentricities tested in the first experiment, to determine whether the increase in heading detection thresholds with retinal eccentricity was a result of decreased acuity at the farthest eccentricities tested. Although static acuity increased with retinal eccentricity, an ANCOVA indicated that the effect of retinal eccentricity on heading thresholds-considered independently with regard to acuitywas significant.

In a third experiment, we derived heading thresholds and Vernier acuity thresholds. The results of this experiment were similar to the results of the first and second experiment: Accuracy in heading judgments decreased with an increase in retinal eccentricity, when controlled for acuity.

Finally, we compared the results of Experiments 1 and 3 with recent results (Atchley \& Andersen, 1998) on sensitivity to radial motion at different retinal eccentricities. The results for sensitivity to radial motion, as a function of retinal eccentricity, were quite similar to the results for heading thresholds at the same eccentricities. A correlation of the two measures indicated that the results for radial motion sensitivity are highly predictive of the results for heading thresholds.

The combined results suggest that central vision is particularly well suited for the use of expansion information present in optic flow fields in detecting heading, relative to objects in the environment, and that the discrimination of heading from optic flow is not as precise in the retinal periphery. Cutting et al. (1992) provide an analysis of the degree of precision necessary to accomplish wayfinding on foot, using FOE information. They suggest that heading accuracy should be $2^{\circ}$ or less for accurate locomotion. In our experiments, this limit was exceeded at all retinal eccentricities except the $0^{\circ}$ condition. In addition, an analysis of the effect size $\left(\omega^{2}\right)$ of the ANCOVA results indicated that the variance accounted for by all three significant pairwise comparisons in Experiment 1 exceeded criteria for a large effect (see, e.g., Cohen, 1977, pp. 284-288). We therefore conclude that the use of radial flow information for determining heading relative to a target is significantly different in central vision than in more peripheral regions of the visual field. These results suggest that the functional sensitivity hypothesis is a valid description of the nature of the human visual system's ability to use radial flow information for determining heading, relative to a target.

Converging evidence for this view has been recently suggested by neurophysiological studies. Early work by Duffy and Wurtz (1991a, 1991b, 1993) suggested that certain areas of the primate visual cortex (MSTd) are 
sensitive to the expansion information of the type present in optic flow. More recently, Duffy and Wurtz (1995) provided neurophysiological evidence suggesting that sensitivity to radial flow should vary as a function of retinal eccentricity. In their experiments, they stimulated 245 neurons in MSTd and found that $90 \%$ of those neurons responded selectively to radial flow (expansion or contraction) located in one part of the visual field. When the center of radial flow was shifted away from that location, most of the neurons exhibited different patterns of activation. A map of the location of these expansion detectors across a horizontal axis of visual space revealed that the greatest concentration of these neurons was in the center of the visual field.

The results of Duffy and Wurtz (1995) and the results of the present experiments suggest that presentation of radial flow information specifying heading to the central visual field may yield lower heading detection thresholds because it has a higher spatial sensitivity for detecting that type of information. In the central visual field, a small change in the location of the FOE is likely to activate another radial flow sensitive neuron because of the higher concentration of these receptors in the central visual field. In the peripheral visual field, the probability of the FOE falling upon a radial flow sensitive neuron is lower because of the decrease in the number of radial flow sensitive neurons in the peripheral visual field. Therefore, larger spatial changes in the FOE are necessary in the peripheral visual field before a change in the FOE can be detected.

Crowell and Banks $(1993,1996)$ argued that changes in performance on psychophysical tasks, such as changes in the detection of heading from optic flow with changes in viewing eccentricity, might be due to two possibilities. First, the amount of information available in the internal representation of the organism may be changing. Such changes might be due, for example, to decreased numbers of detectors tuned to the relevant characteristic of the flow field, such as the FOE in the case of heading detection. The second possibility is that the same amount of information is available but the relative efficiency with which the information is processed changes. Crowell and Banks (1996) constructed an ideal-observer model for heading judgments to assess these possibilities. Efficiency is defined as the ratio of the ideal-observer threshold to the human threshold. Whereas they found that, overall, efficiency for the detection of heading generally increased with retinal eccentricity, for radial flow patterns efficiency was highest at $0^{\circ}$. Furthermore, this effect is suggestive of specialized neural mechanisms for processing radial flow in the central visual field, which is consistent with the results of psychophysical experiments and the data of Duffy and Wurtz (1995).

The results of the present study, considered with the results of other heading studies, suggest that different information is used in different regions of the visual field. The present results indicate that determining heading from radial flow is optimal in the central visual field. In addition, DMP - which is dependent on information immediately surrounding the point of fixation-provides heading information to the central visual field (Cutting, 1986; Cutting et al., 1992). In contrast, asymmetric flow fields provide useful information for heading in either the central field or the peripheral visual field (Dyre \& Andersen, 1997; see, also, Sauvan \& Bonnet, 1993). Thus, the visual system has available multiple sources of information for heading that are particularly useful in different regions of the visual field.

In conclusion, the results of the present study indicate that the discrimination of heading from optic flow is not retinally invariant. Heading detection thresholds were significantly lower in the central vision field than in more peripheral regions of the visual field. Two additional experiments and the use of an ANCOVA discounted the possibility that changes in acuity were responsible for these results. The current results suggest that sensitivity to radial flow for determining heading relative to a target varied as a function of retinal eccentricity. This finding reflects the specialized role of central vision for determining heading from radial flow.

\section{REFERENCES}

Andersen, G. J., \& Braunstein, M. L. (1985). Induced self-motion in central vision. Journal of Experimental Psychology: Human Perception \& Performance, 11, 122-132.

ANdersen, G. J., \& Dyre, B. P. (1989). Spatial orientation from optic flow in the central visual field. Perception \& Psychophysics, 45, 453-458.

ATCHLEY, P., \& ANDERSEN, G. J. (1998). The effect of age, retinal eccentricity, and speed on the detection of optic flow components. Psychology \& Aging, 13, 297-308.

Brandt, T., Dichgans, J., \& KoEnig, E. (1973). Differential effects of central versus peripheral vision on egocentric and exocentric motion perception. Experimental Brain Research, 16, 476-491.

BROWN, B. (1972). The effect of target contrast variation on dynamic visual acuity and eye movements. Vision Research, 12, 1213-1224.

COHEN, J. (1977). Statistical power analysis for the behavioral sciences. New York: Academic Press.

Crowell, J. A., \& Banks, M. S. (1993). Perceiving heading with different retinal regions and types of optic flow. Perception \& Psychophysics, 53, 325-337.

Crowell, J. A., \& Banks, M. S. (1996). Ideal observer for heading judgments. Vision Research, 36, 471-490.

Cutting, J. E. (1986). Perception with an eye for motion. Cambridge, MA: MIT Press.

Cutting, J. E. (1996). Wayfinding from multiple sources of local information in retinal flow. Journal of Experimental Psychology: Human Perception \& Performance, 22, 1299-1313.

Cutting, J. E., Springer, K., Braren, P. A., \& Johnson, S. H. (1992). Wayfinding on foot from information in retinal, not optical, flow. Journal of Experimental Psychology: General, 121, 41-72.

Cutting, J. E., Vishton, P. M., Flückiger, M., Baumberger, B., \& GERNDT, J. D. (1997). Heading and path information from retinal flow in naturalistic environments. Perception \& Psychophysics, 59, 426-441.

Duffy, C. J., \& WuRTZ, R. H. (1991a). Sensitivity of MST neurons to optic flow stimuli: I. A continuum of response selectivity to largefield stimuli. Journal of Neurophysiology, 65, 1329-1345.

DufFY, C. J., \& WuRTZ, R. H. (1991b). Sensitivity of MST neurons to optic flow stimuli: II. Mechanisms of response selectivity revealed by small-field stimuli. Journal of Neurophysiology, 65, 1346-1359. 
DuFFY, C. J., \& WuRTz, R. H. (1993). An illusory transformation of optic flow fields. Vision Research, 33, 1481-1490.

DuFFy, C. J., \& WURTZ, R. H. (1995). Response of monkey MST neurons to optic flow stimuli with shifted centers of motion. Journal of Neuroscience, 15, 5192-5208.

Dyre, B. P., \& ANDERSEN, G. J. (1997). Perception of heading: Effects of conflicting velocity magnitude and trajectory information. Journal of Experimental Psychology: Human Perception \& Performance, 23, 546-565.

GiBson, J. J. (1950). The perception of the visual world. Boston: Houghton Mifflin.

GIBson, J. J. (1966). The senses considered as perceptual systems. Boston: Houghton Mifflin.

HELD, R. (1970). Two modes of processing spatially distributed visual stimulation. In F. O. Schmidt (Ed.), The neurosciences: Second study (pp. 317-323). New York: Rockefeller University Press.

Held, R., DichGaNs, J., \& Bauer, J. (1975). Characteristics of moving visual scenes influencing spatial orientation. Vision Research, 15, 357-365.

KEPPEL, G. (1982). Design and analysis. Engelwood Cliffs, NJ: PrenticeHall.

Leibowitz, H. W., Johnson, C. A., \& Isabelle, E. (1972). Peripheral motion detection and refractive error. Science, 177, 1207-1208.

LEVI, D. M., KLEIN, S. A., \& AitsEBAOMO, A. P. (1985). Vernier acuity, crowding, and cortical magnification. Vision Research, 25, 963-977.

SaUVAN, X. M., \& Bonnet, C. (1993). Properties of curvilinear vection. Perception \& Psychophysics, 53, 429-435.
Stoffregen, T. A. (1985). Flow structure versus retinal location in the optical control of stance. Journal of Experimental Psychology: Human Perception \& Performance, 11, 554-565.

StofFreGen, T. A., \& Riccio, G. E. (1990). Responses to optical looming in the retinal center and periphery. Ecological Psychology, 2, 251-274.

WARREN W. H. (1995). Self-motion: Visual perception and visual control. In W. Epstein \& S. Rogers (Eds.), Perception of space and motion (pp. 263-326). San Diego: Academic Press.

WARREN, W. H., \& HANNON, D. J. (1990). Direction of self motion from optic flow. Nature, 336, 162-163.

WARREN, W. H., \& KURTZ, K. J. (1992). The role of central and peripheral vision in perceiving the direction of self-motion. Perception \& Psychophysics, 51, 443-454.

Warren, W. H., Morris, M. W., \& Kalish, M. (1988). Perception of translational heading from optical flow. Journal of Experimental Psychology: Human Perception \& Performance, 14, 646-660.

\section{NOTE}

1. The target bar was presented in the center of the display, because we varied retinal eccentricity by repositioning the monitor relative to the observer, instead of repositioning the bar relative to the screen.

(Manuscript received February 6, 1997; revision accepted for publication March 18, 1998.) 